Research Article

\title{
Nonlinear Vibration Analysis of Submerged Floating Tunnel Tether
}

\author{
Sheng-nan Sun $\mathbb{D}$, Zhi-bin Su $\mathbb{D}$, and Yun-fen Feng \\ School of Architecture and Civil Engineering, Liaocheng University, Liaocheng 252059, China \\ Correspondence should be addressed to Sheng-nan Sun; sunshengnan@lcu.edu.cn
}

Received 27 March 2020; Revised 18 November 2020; Accepted 5 December 2020; Published 19 December 2020

Academic Editor: Evangelos J. Sapountzakis

Copyright (c) 2020 Sheng-nan Sun et al. This is an open access article distributed under the Creative Commons Attribution License, which permits unrestricted use, distribution, and reproduction in any medium, provided the original work is properly cited.

\begin{abstract}
To study the nonlinear vibration characteristics of submerged floating tunnel tether, the nonlinear vibration partial differential equation of tether is set up, in which the inclination, sag, initial tension force, and length of tether are taken into account. According to the linear vibration mode of tether, the partial differential equation is converted into ordinary differential equations by Galerkin method and the nonlinear vibration equation of tether for the in-plane first four modes and the out-of-plane first four modes are obtained. The results show that the change of inclination results is in the change of sag. The initial configuration of sag is symmetric. Thus, the sag only affects the damping ratio of the in-plane symmetric mode, namely, that of the first and the third mode. The sag has no effect on the second and forth order modes which are antisymmetric modes. Therefore, the sag is only existed in plane and has no effect on the out-of-plane mode. The first and the third in-plane modal damping ratios of tether are in direct proportion to their inclination, whereas in inverse proportion to their sag. The first modal damping ratio of tether (both inplane and out-of-plane) is in direct proportion to its length, whereas in inverse proportion to its initial tension.
\end{abstract}

\section{Introduction}

Submerged floating tunnel (also known as Archimedes bridge) is a new type of structure, which can span different types of waters, such as bays, rivers, straits, and lakes. [1]. It is different from any other tunnel engineering in the past and has a unique anchorage system. While improving the traffic condition, the impact of submerged floating tunnel on the surrounding environment is very limited, which will not damage the natural landscape of the construction site and affect the ground traffic (while improving traffic conditions, the submerged floating tunnel will have a very limited impact on the surrounding environment and will not damage the natural landscape [2] of the site or affect surface traffic).

Tethers are the most important components of submerged floating tunnels. They keep the structure stable against various environment loads such as wave, current, and earthquake. Submerged floating tunnel tethers are light, very flexible, and lightly damped, which make them particularly prone to vibrate [3-5].
Vibration of tether was studied by many experts and scholars. Ge et al. [6] investigated the effect of tunnel surge motion on the dynamic response of a tether by considering the forcing and parametric excitations. Chen et al. [7], taking submerged floating tether as a beam subjected to tension, set up vortex-induced oscillation equations of tether and discussed effects of tether's obliquity, tension, and length on its maximal dynamic shearing force and bending moments. Ge et al. [8] investigated vortex-induced vibration of submerged floating tunnel tube-tether coupled model. Considering nonlinear coupling effect between the axial and transverse vibrations of tether, the dynamic response model of submerged floating tunnel was presented. Luo et al. [9], considering the nonlinear characteristics of submerged floating tunnel tether and the lateral dynamic response of structurefluid interaction, established a nonlinear dynamic model equation of tether and solved it by the secondary development of finite element software. To solve the dynamic response of submerged floating tunnel under current, Xiang and Chao [10] studied the vortex-induced vibration and parametric vibration of tube and tethers. 
There are also many works about uniform/nonuniform risers in the linear and nonlinear ranges. Caruntu [11] studied the primary resonance of single-mode forced undamped bending vibration of nonuniform sharp cantilevers which are rectangular cross sections and had constant width and convex parabolic thickness variation. Arefi and Zenkour [12] studied thermoelectromechanical transient analysis of a sandwich nanoplate. Guo and Yang [13] investigated free longitudinal vibrations of nonuniform rods by a proposed method, which results in a series solution. Arefi and Zenkour [14] studied wave propagation analysis of a nanobeam made of functionally graded magnetoelectroelastic materials with rectangular cross-section rest on Visco-Pasternak's foundation. Lenci et al. [15] used the asymptotic development method to obtain approximate analytical expressions for the natural frequencies of nonuniform cables and beams. Arefi and Zenkour [16] studied size-dependent free vibration analysis of a sandwich nanoplate. Bambill et al. [17] used the differential quadrature method and the domain decomposition to carry out the free transverse vibration analysis of nonuniform multispan rotating Timoshenko beams with perfect and not perfect boundary conditions. Zenkour and Arefi [18] developed the transient thermoelectromechanical vibration and bending analysis of a functionally graded piezoelectric nanosheet rest on Visco-Pasternak's foundation. Babilio [19] considered the dynamics of a simply supported beam which is made of an axially functionally graded material under axial time-dependent load. Arefi and Zenkour [20] studied wave propagation analysis for a functionally graded nanobeam with rectangular cross-section resting on Visco-Pasternak's foundation. Clementi et al. [21] determined the frequency response curves of a nonuniform beam undergoing nonlinear oscillations analytically by the multiple time scale method, which provides approximate but accurate results. Arefi and Zenkour [22] expressed magnetoelectrothermomechanical bending and free vibration analysis of a sandwich microplate using strain gradient theory. Babilio and Enrico [23] studied the nonlinear dynamics of beams under axial time-dependent excitation.

In this paper, to study the nonlinear vibration characteristics of submerged floating tunnel tether, the nonlinear vibration partial differential equation of tether is set up, in which the inclination, sag, initial tension force, and length of tether are taken into account. According to linear vibration mode of tether, the partial differential equation is converted into ordinary differential equations by the Galerkin method and the nonlinear vibration equation of tether for the inplane first four modes and the out-of-plane first four modes are obtained.

\section{Nonlinear Free Vibration Model of Tether}

2.1. Vibration Equation. Figure 1 shows the nonlinear analytical model proposed for the vibration analysis of tether. Let $\boldsymbol{Z}=\left[\begin{array}{lll}x(s) & y(s)\end{array}\right]^{\mathrm{T}}$ denote the static configuration of tether, $s$ being a curvilinear abscissa. The dynamic configuration subjected to dynamic loading is described by the displacement $\boldsymbol{U}=[u(z, t) v(z, t)]^{\mathrm{T}}$ measured from the

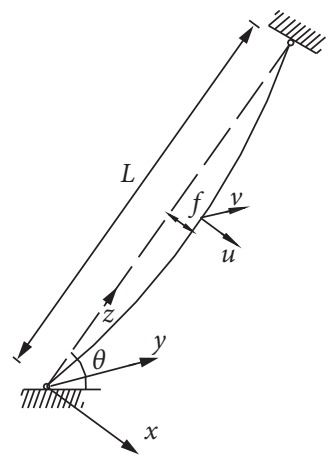

FIgURE 1: Nonlinear free vibration model of tether.

static equilibrium position. $t$ is time and $x, y$, and $z$ are Cartesian coordinates, as shown in Figure 1.

The governing differential equations of the tether motion can be derived using the Hamilton principle [24]:

$$
\delta \int_{t_{1}}^{t_{2}} \int_{0}^{L}(Q-V) \mathrm{d} s \mathrm{~d} t+\int_{t_{1}}^{t_{2}} \int_{0}^{L} \delta W \mathrm{~d} s \mathrm{~d} t=0,
$$

in which $\delta$ is the symbol for variation; $t_{1}$ and $t_{2}$ are the limits of the time interval; $L$ is the unstretched length of tether; and $\mathrm{d} t$ is the differential of time.

$Q$ is the kinetic energy density [24]:

$$
Q=\frac{m}{2}(\dot{U})^{T} \dot{U}
$$

$V$ is the elastic strain energy density [24]:

$$
V=V_{i}+\frac{\mathrm{EA}}{2} \varepsilon^{2}+T_{0} \cdot \varepsilon
$$

$\varepsilon$ is the Lagrangian strain [24]:

$$
\varepsilon=\left(Z^{\prime} Z^{\prime}\right)^{T} U^{\prime}+\frac{1}{2}\left(U^{\prime}\right)^{T} U^{\prime}
$$

$\delta W$ is the virtual work density associated with the gravity, dynamic loading, and damping force [24]:

$$
\delta W=\delta \mathbf{U}^{T}\left[\gamma_{f} V_{S} \cos \theta-\mathbf{c} \dot{U}+F_{D}\right],
$$

where $E, A$, and $m$ denote the modulus of elasticity, the cross-sectional area, and the mass per unit length of unstretched tether, respectively; $V_{\mathrm{i}}$ is the elastic strain energy density held in the initial (static) configuration; $T_{0}$ is the tether tension in the static configuration; $\gamma_{\mathrm{f}}$ is the buoyant unit weight of tether; $V_{\mathrm{S}}$ is the volume per unit length of unstretched tether; $\theta$ is the tether inclination with respect to the horizontal level; $c$ is the viscous damping coefficient of tether, $\mathbf{c}=\operatorname{Diag}\left[\begin{array}{ll}c_{x} & c_{y}\end{array}\right]$; and $F_{\mathrm{D}}$ is the acting force applied by water per unit length when the tether vibrates.

We assume that the tether to be considered here has small sag, i.e., the ratio of static displacement in the middle of the tether $f$ to the unstretched length of tether $L$ ranging from nearly zero to about $1 / 8$. The static equilibrium position of the tether can be approximated by the quadratic parabola: 


$$
x=4 f \frac{z}{L}\left(1-\frac{z}{L}\right)
$$

By substituting equations (2)-(6) into equation (1), omitting balance items under static loads, higher order small amount, and small amount of $z$-direction motion, the following nonlinear partial differential equation is obtained. Since the bending stiffness of the tether is very small, it is ignored. Meanwhile, considering that the initial tension force of tether is far greater than its own gravity, the change of tension along the length is ignored:

$$
\begin{gathered}
m \frac{\partial^{2} u}{\partial t^{2}}+c_{x} \frac{\partial u}{\partial t}-E A \frac{\partial}{\partial z}\left(\varepsilon \frac{\partial x}{\partial z}\right)-T_{0} \frac{\partial^{2} u}{\partial z^{2}}=-F_{D}, \\
m \frac{\partial^{2} v}{\partial t^{2}}+c_{y} \frac{\partial v}{\partial t}-T_{0} \frac{\partial^{2} v}{\partial z^{2}}=-F_{D}, \\
\varepsilon=\frac{\gamma_{f} V_{s} \cos \theta}{H_{0} L_{E}} \int_{0}^{L} u \mathrm{~d} z \\
L_{E}=L\left[1+\left(\frac{f}{L}\right)^{2}\right] \\
f=\frac{\gamma_{f} V_{s} L^{2} \cos \theta}{\left(8 H_{0}\right)},-F_{\mathrm{D}}
\end{gathered}
$$

in which $H_{0}$ is the $z$-direction component of tension and $T_{0} \approx H_{0} ; L_{\mathrm{E}}$ is the tether length in the static configuration; and $f$ is the static displacement in the middle of tether.

Equations (7) and (8) are in-plane vibration equation and out-of-plane vibration equation of tether, respectively. Due to these two equations being similar, here only equation (7) is analyzed in detail.

According to Morison equation, when the tether vibrates, the total acting force induced by water per unit length may be expressed as the sum of the damping force and the additional inertial force [25]:

$$
F_{D}=\frac{\pi D_{t}^{2}}{4} \rho_{w} C_{m} \ddot{u}+\frac{1}{2} \rho_{w} D_{t} C_{D} \dot{u}|\dot{u}|
$$

where $D_{\mathrm{t}}$ is the diameter of the tether; $\rho_{\mathrm{w}}$ is the water density and $\rho_{\mathrm{w}}=1028 \mathrm{~kg} / \mathrm{m} ; C_{\mathrm{m}}$ is the additional mass coefficient and $C_{\mathrm{m}}=1$; and $C_{\mathrm{D}}$ is the drag force coefficient and $C_{\mathrm{D}}=0.7$.

When the mode order calculated increases, numerical calculation becomes more difficult due to the nonlinearity of $1 / 2 \rho_{\mathrm{w}} D_{\mathrm{t}} C_{\mathrm{D}} \dot{u}|\dot{u}|$ in equation (12). Therefore, here linearized Morison equation is adopted [26]:

$$
F_{\mathrm{D}}=\frac{1}{2} C_{\mathrm{D}} \rho_{\mathrm{w}} D_{\mathrm{t}} \sqrt{\frac{8}{\pi}} \sigma_{\dot{u}} \dot{u}+C_{\mathrm{m}} \frac{\pi D_{\mathrm{t}}^{2}}{4} \rho_{\mathrm{w}} \ddot{u}
$$

in which $\sigma_{\dot{u}}$ is the variance of $\dot{u}$.

Finally, equation (7) may be written as

$$
\begin{aligned}
- & T_{0} \frac{\partial^{2} u}{\partial z^{2}}+\bar{m} \frac{\partial^{2} u}{\partial t^{2}}+c_{x} \frac{\partial u}{\partial t}+E A \frac{8 f}{L^{2}} \frac{\gamma_{f} V_{s} \cos \theta}{T_{0} L_{E}} \int_{0}^{L} u d z \\
+ & \frac{\rho_{w}}{2} C_{D} \cdot D_{t} \sqrt{\frac{8}{\pi}} \frac{\partial u}{\partial t} \sigma_{\dot{u}}=0,
\end{aligned}
$$

where $\bar{m}$ is the sum of the tether mass and added mass per unit unstretched length.

By separation of variables, vibration mode of tether may be expressed as

$$
u(z, t)=\sum_{n=1}^{N} u_{n}(t) \sin \frac{n \pi z}{L} .
$$

By means of the Galerkin method, the partial differential equation is transformed into a set of ordinary ones

$$
[\mathbf{M}] \ddot{u}+[\mathbf{C}] \dot{u}+[\mathbf{K}] u=0,
$$

$$
\begin{aligned}
{[\mathbf{M}] } & =\left[\mathbf{M}_{1}\right]+\left[\mathbf{M}_{2}\right], \\
{[\mathbf{C}] } & =\left[\mathbf{C}_{1}\right]+\left[\mathbf{C}_{2}\right], \\
{[\mathbf{K}] } & =\left[\mathbf{K}_{1}\right]+\left[\mathbf{K}_{2}\right],
\end{aligned}
$$

where $\left[\boldsymbol{M}_{1}\right]$ and $\left[\boldsymbol{M}_{2}\right]$ are mass matrix and added mass matrix of tether, respectively; $\left[\boldsymbol{C}_{1}\right]$ and $\left[\boldsymbol{C}_{2}\right]$ are damping matrix of tether and damping matrix provided by water, respectively; and $\left[\boldsymbol{K}_{1}\right]$ and $\left[\boldsymbol{K}_{2}\right]$ are equivalent stiffness matrix provided by initial tension and dynamic tension, respectively.

2.2. Solution of Equation. Suppose that the solution of equation (14) may be written as the following form:

$$
u_{j}=A_{j} \mathrm{e}^{\eta t} \quad(j=1,2, \ldots, n) .
$$

Substituting equation (16) into equation (14) and eliminating common factors, the following equation is obtained:

$$
\left(\eta^{2}[\mathbf{M}]+\eta[\mathbf{C}]+[\mathbf{K}]\right)\{\mathbf{u}\}=0,
$$

in which $\{\boldsymbol{u}\}=\left[u_{1}, u_{2}, \ldots, u_{n}\right]^{\mathrm{T}}$.

Introducing the following identity

$$
[\mathbf{M}] \dot{u}-[\mathbf{M}] \dot{u}=0,
$$

equations (18) and (14) make up a simultaneous equation which is written as

$$
\mathbf{A} \dot{q}+\mathbf{B} q=0
$$

in which $\mathbf{A}=\left[\begin{array}{cc}{[0]} & {[\mathbf{M}]} \\ {[\mathbf{M}]} & {[\mathbf{C}]}\end{array}\right], \quad \mathbf{B}=\left[\begin{array}{cc}-[\mathbf{M}] & {[0]} \\ {[0]} & {[\mathbf{K}]}\end{array}\right], \quad$ and $q=\left\{\begin{array}{l}\dot{u} \\ u\end{array}\right\}$.

Let $q=\{v\} \mathrm{e}^{\eta t}$ and substituting $q$ into equation (19), the following equation is obtained:

$$
(\eta \mathbf{A}+\mathbf{B})\{v\}=0 .
$$

When $\operatorname{det}|\eta \boldsymbol{A}+\boldsymbol{B}|=0$, equation (20) has a nonzero solution. Solving it by two-step QR algorithm, $2 n$ conjugate complex eigenvalues of the tether system may be obtained. Real part and imaginary part of the ith pair complex eigenvalue correspond to modal damping ratio and frequency of the $i$ th order mode of system, respectively: 
TABLE 1: Basic parameters of tether.

\begin{tabular}{lc}
\hline Parameter & Value \\
\hline Length $(\mathrm{m})$ & 161.66 \\
Diameter $(\mathrm{m})$ & 0.489 \\
Mass per unit length $(\mathrm{kg} / \mathrm{m})$ & 1474.23 \\
Added mass per unit length $(\mathrm{kg} / \mathrm{m})$ & 193.06 \\
Density $\left(\mathrm{kg} / \mathrm{m}^{3}\right)$ & 7850 \\
Elastic modulus $(\mathrm{Pa})$ & $2.1 \times 10^{11}$ \\
Initial disturbance $(\mathrm{m})$ & 0.01 \\
Initial tension force $(\mathrm{N})$ & $2.572 \times 10^{7}$ \\
Damping ratio & 0.0018 \\
\hline
\end{tabular}

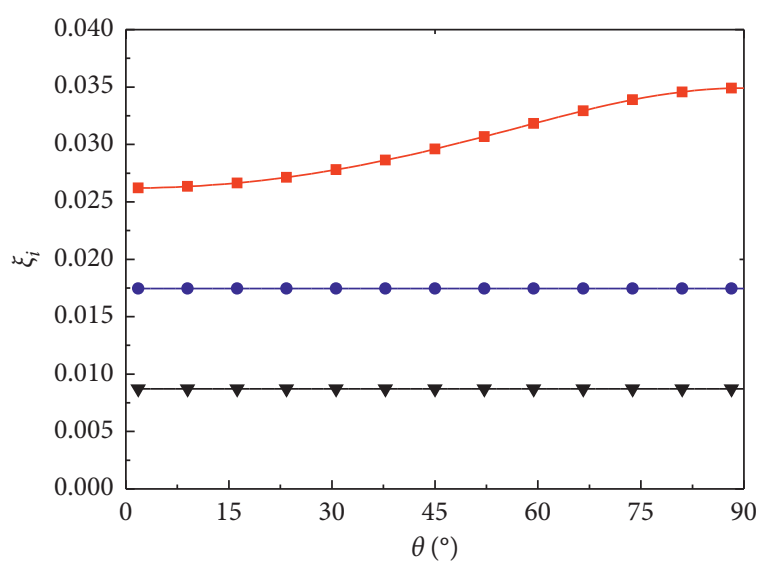

- First order mode

- Second order mode

$\rightarrow$ Fourth order mode

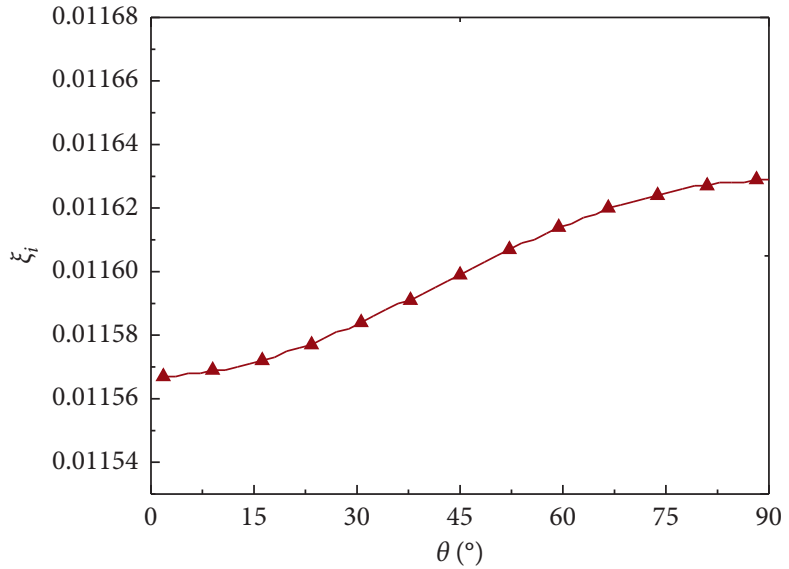

— Third order mode

FIgURE 2: Relationship between in-plane modal damping ratios and inclination of tether.

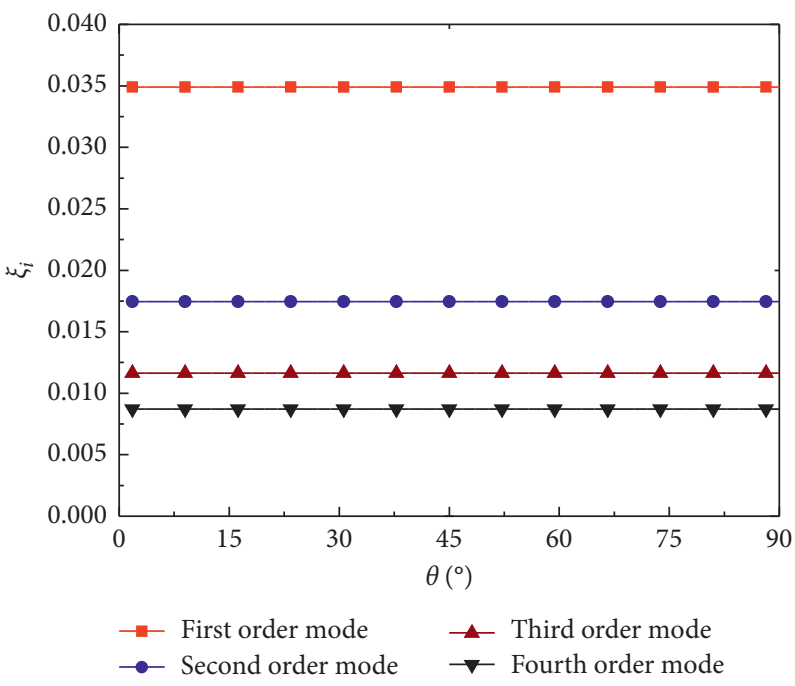

FIGURE 3: Relationship between out-of-plane modal damping ratios and inclination of tether. 


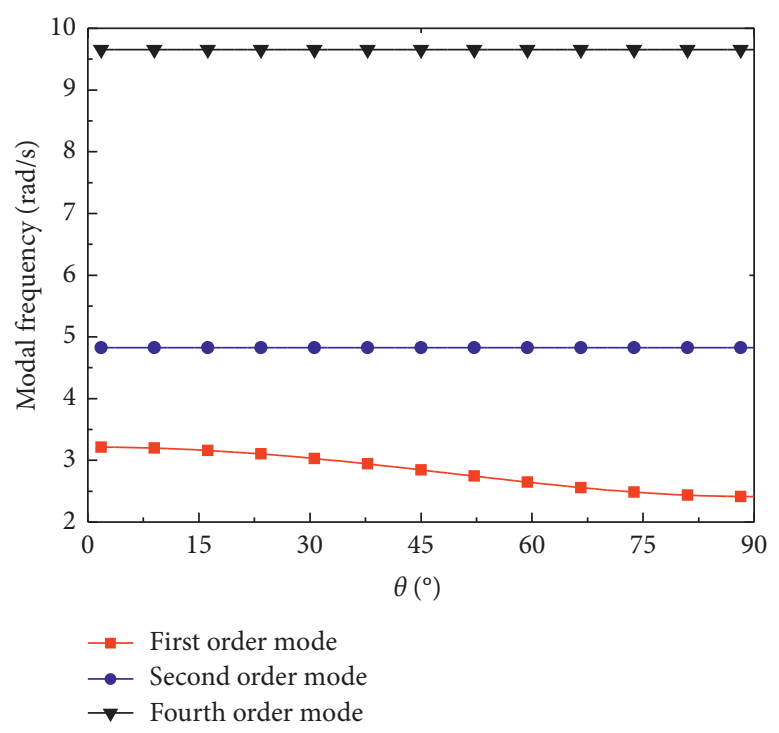

(a)

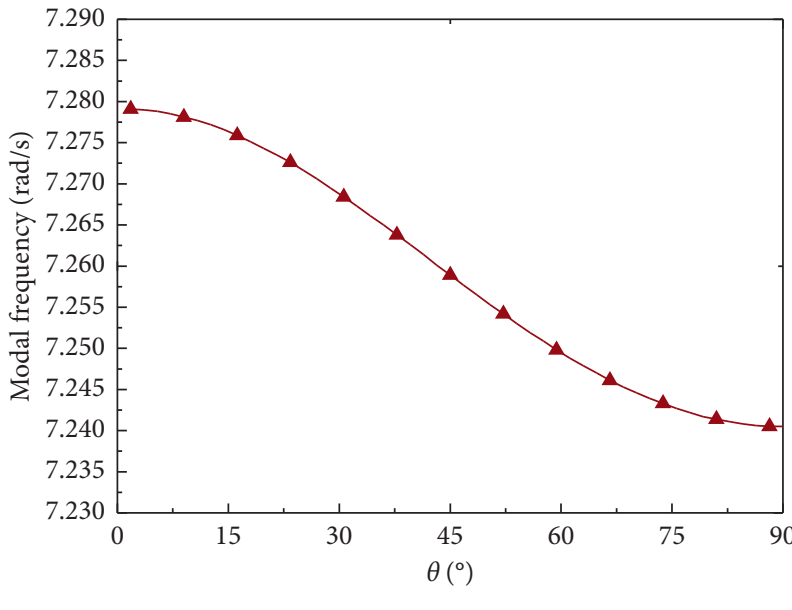

^ Third order mode

Figure 4: Relationship between in-plane modal frequency and inclination of tether.

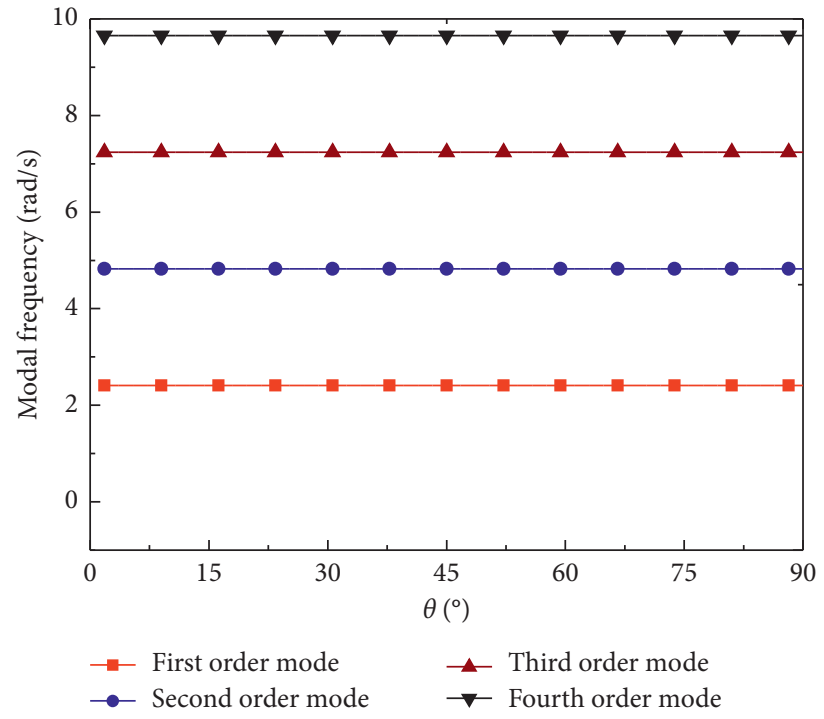

FIGURE 5: Relationship between out-of-plane modal frequency and inclination of tether.

$$
\eta_{i}=\omega_{i}\left(-\xi_{i} \pm \sqrt{-1} \sqrt{1-\xi_{i}^{2}}\right)
$$

For solving of out-of-plane vibration equation (8), its process is similar to that of equation (7) except $\left[\boldsymbol{K}_{2}\right]=0$. Based on the abovementioned theory, dynamic solving program of nonlinear free vibration system is worked out by MATLAB and numerical analysis is presented.

\section{Numerical Analysis}

For the convenience of discussion, the following dimensionless parameter which reflects the sag and tensile property of tether is introduced [27]:

$$
\lambda^{2}=\left(\frac{\gamma_{\mathrm{f}} V_{\mathrm{s}} L \cos \theta}{H_{0}}\right)^{2} \cdot \frac{L}{\left[H_{0} \cdot L_{\mathrm{E}} /(E A)\right]} .
$$

Basic parameters of tether are shown in Table 1.

3.1. Effect of Tether Inclination on Its Modal Damping Ratios. When the tether inclination changes from 0 degrees to 90 degrees, its modal damping ratios of the first four modes (both in-plane and out-of-plane) are obtained.

From Figures 2 and 3, it can be seen that when tether inclination increases, the damping ratio of first and third inplane mode increases, whereas the damping ratios of second and fourth in-plane modes and first four out-of-plane modes 


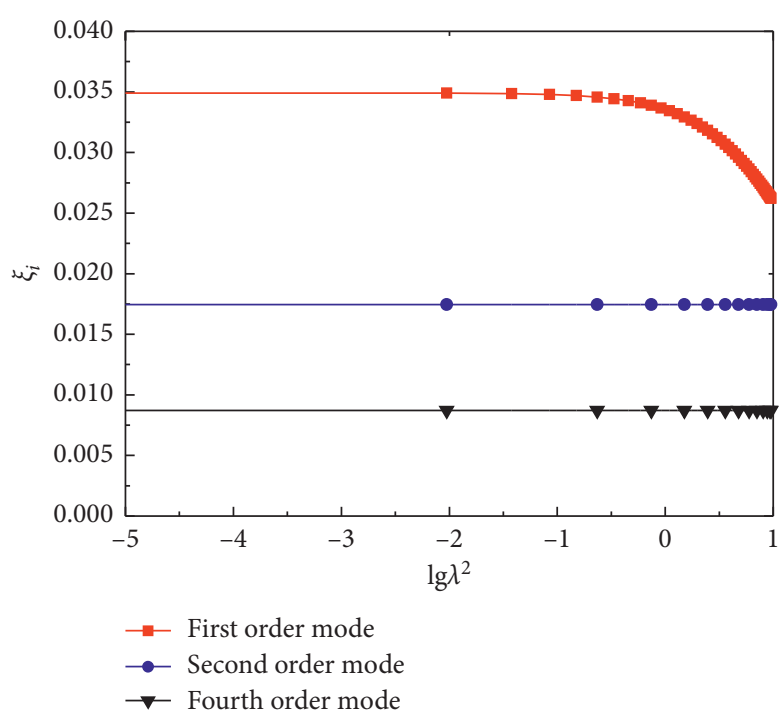

(a)

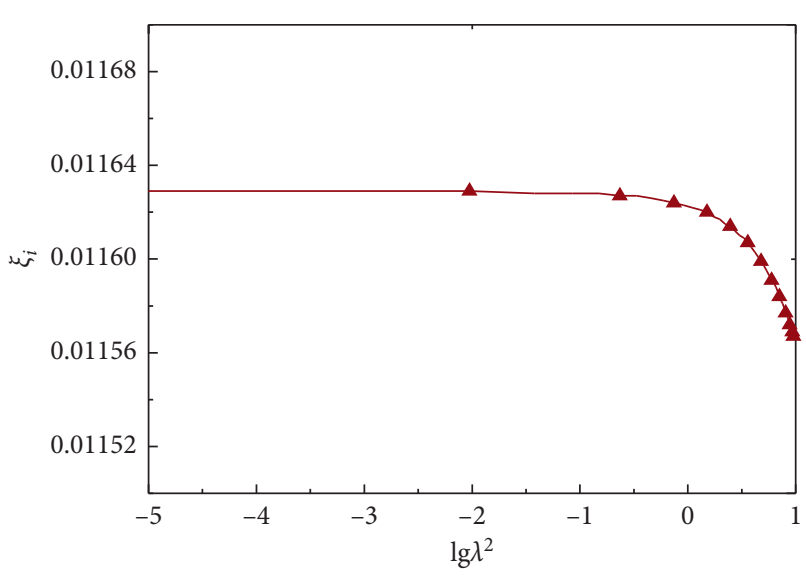

Third order mode

Figure 6: Relationship between in-plane modal damping ratios and sag of tether.

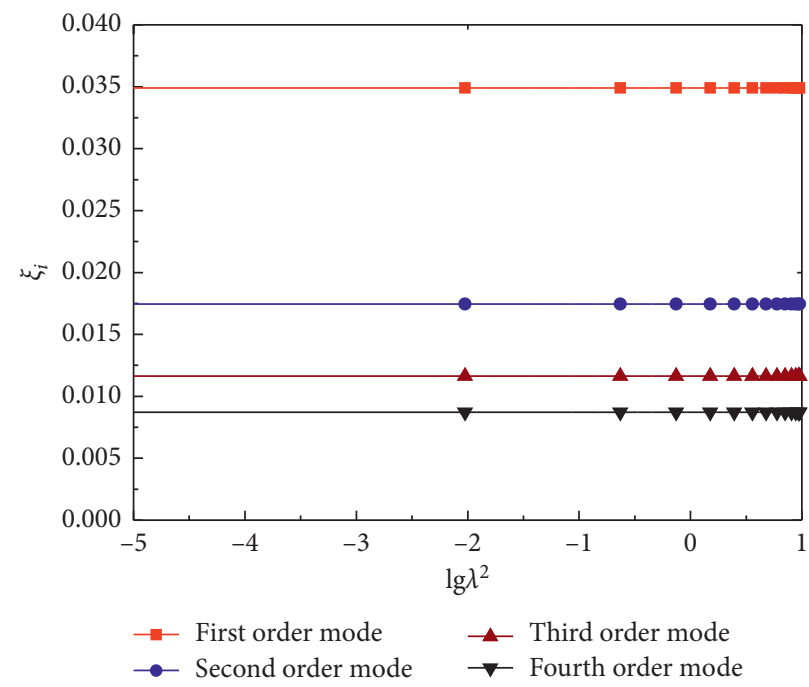

FIgURE 7: Relationship between out-of-plane modal damping ratios and sag of tether.

keep constant. That is to say, tether inclination merely affects the damping ratio of odd order in-plane mode; tether inclination has no effect on the damping ratios of even order in-plane modes and out-of-plane modes.

From Figures 4 and 5, it can be seen that when tether inclination increases, the frequency of first and third inplane mode decreases, whereas the frequency of second and fourth in-plane modes and first four out-of-plane modes keep constant. That is to say, tether inclination merely affects the frequency of odd order in-plane mode; tether inclination has no effect on the frequency of even order in-plane modes and out-of-plane modes.
3.2. Effect of Tether Sag on Its Modal Damping Ratios. Changing sag of tether by changing its inclination, effect of tether sag on its modal damping ratios is shown in Figures 6 and 7. It is shown that when tether sag increases, the damping ratio of first and third in-plane mode decreases, whereas the damping ratios of second and fourth in-plane modes and first four out-of-plane modes keep constant. That is to say, similar to tether inclination, tether sag merely affects the damping ratio of odd order in-plane mode.

From Figures 8 and 9, it can be seen that when tether sag increases, the frequency of first and third in-plane mode increases, whereas the frequency of second and fourth in- 


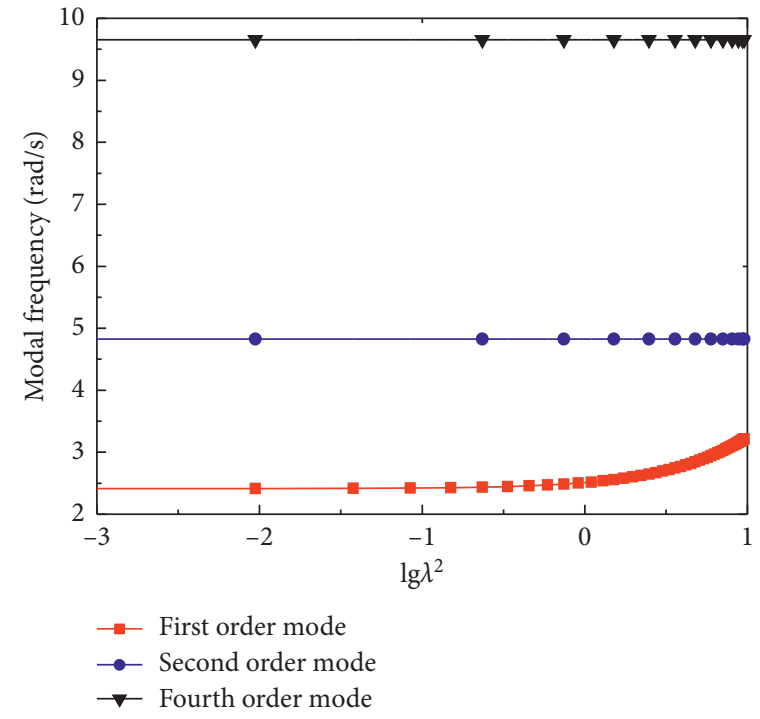

(a)

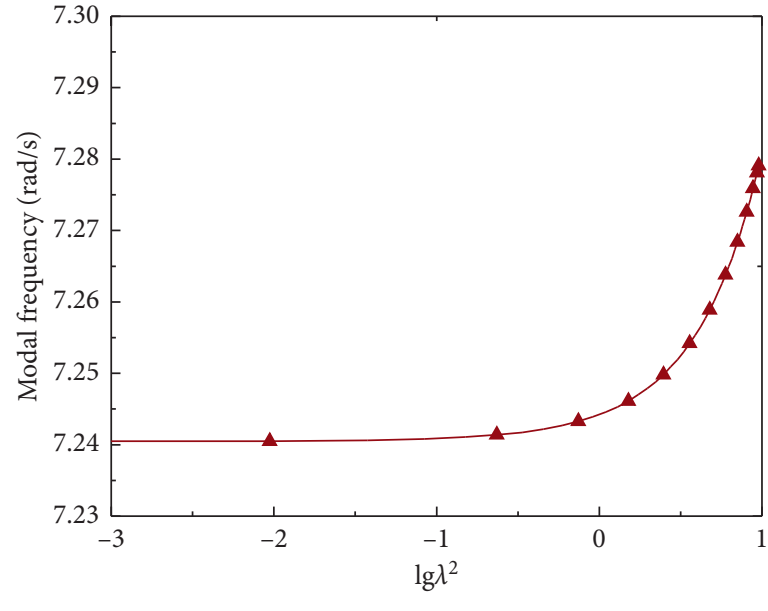

- Third order mode

FIGURE 8: Relationship between in-plane modal frequency and sag of tether.

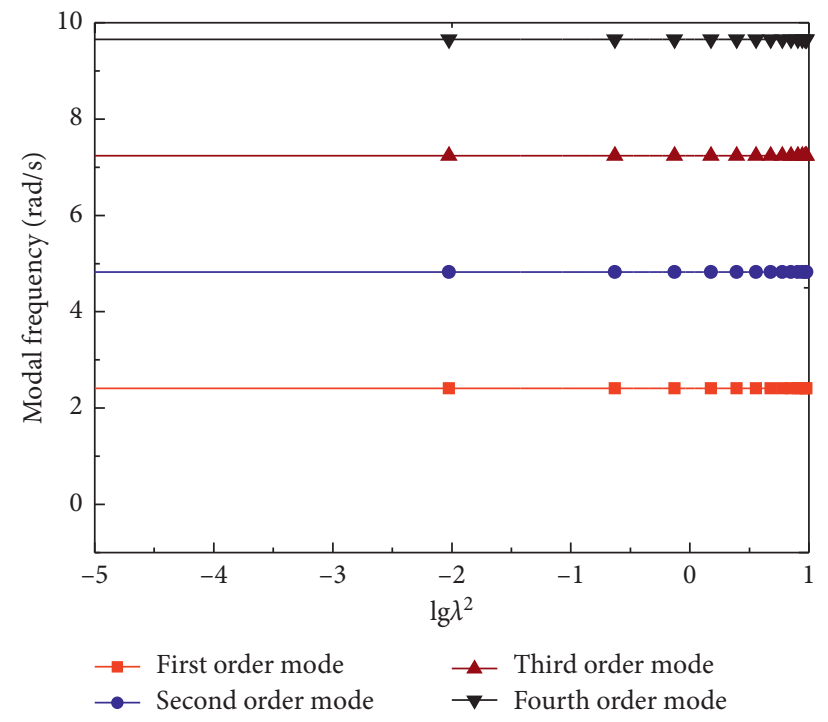

FIGURE 9: Relationship between out-of-plane modal frequency and sag of tether.

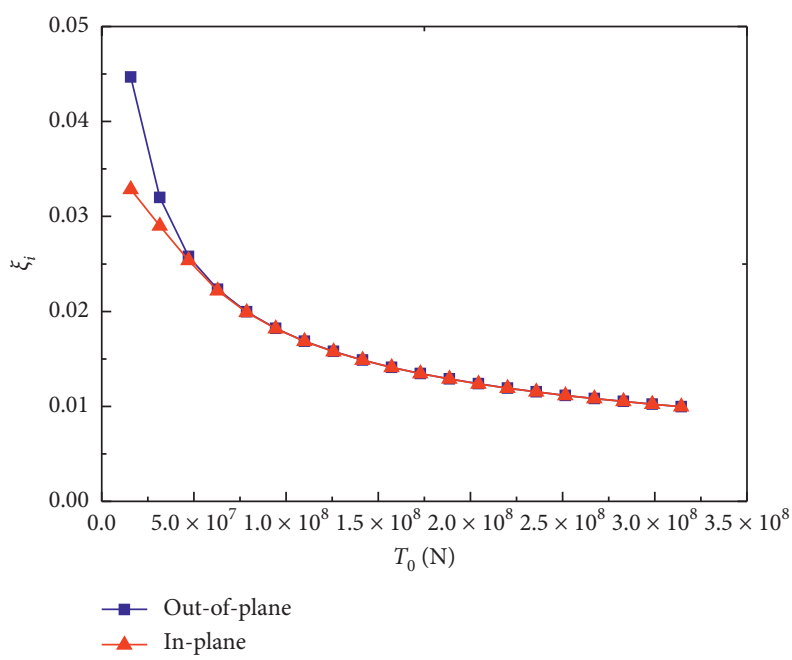

Figure 10: Relationship between modal damping ratios and initial tension of tether. 


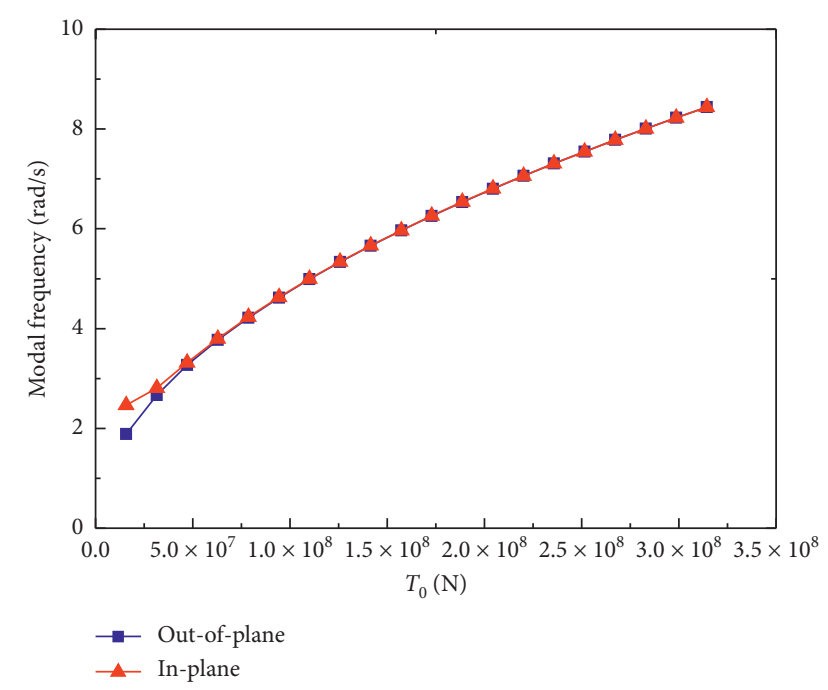

FIGURE 11: Relationship between modal frequency and initial tension of tether.

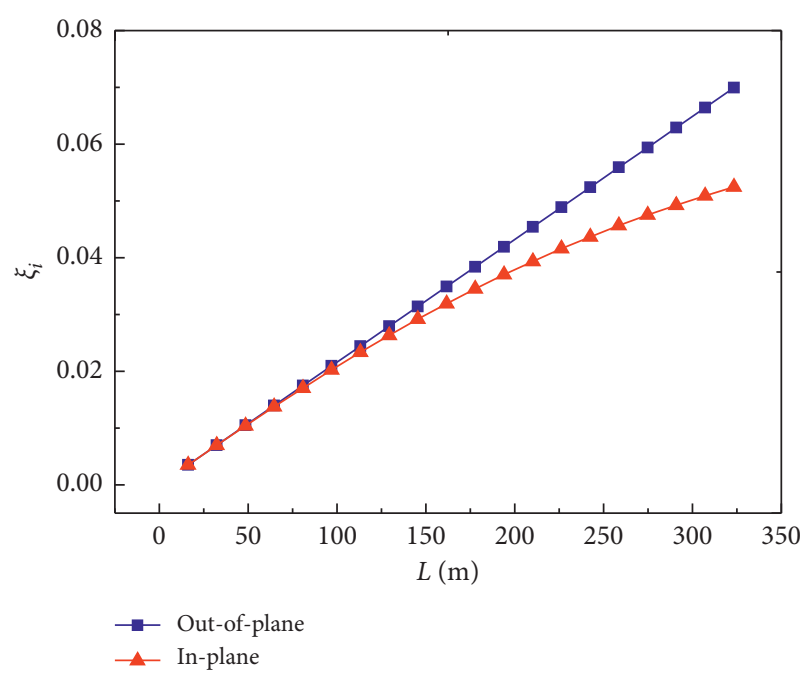

FIGURE 12: Relationship between first order modal damping ratios and length of tether.

plane modes and first four out-of-plane modes keep constant. That is to say, tether sag merely affects the frequency of odd order in-plane mode; tether sag has no effect on the frequency of even order in-plane modes and out-of-plane modes.

The change of inclination leads to the change of sag. The initial configuration of sag is symmetric. Thus, the sag only affects the damping ratio of the inner symmetric mode, namely, that of the first and the third mode. The sag has no effect on the second and forth order modes which are antisymmetric modes. Therefore, the sag is only existed inplane and has no effect on the out-of-plane mode.

3.3. Effect of Tether Tension on Its Modal Damping Ratios. Effect of tether tension on its modal damping ratios of the first mode (both in-plane and out-of-plane) are shown in

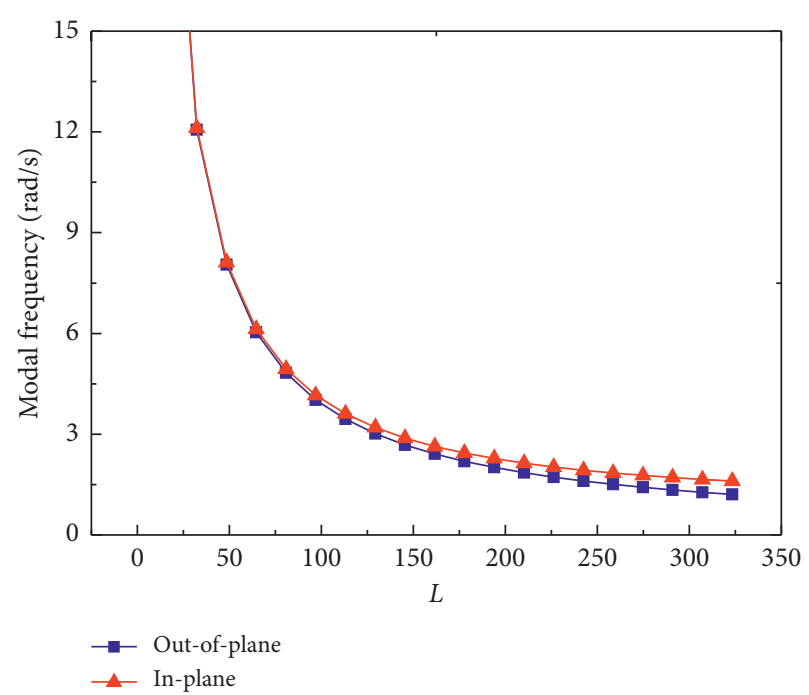

FIGURE 13: Relationship between first order modal frequency and length of tether.

Figure 10. It can be seen that, due to the influence of tether tension on its vibration period, when tether sag increases, the damping ratios of first in-plane and out-of-plane modes decrease.

Effect of tether tension on its modal frequency of the first mode (both in-plane and out-of-plane) are shown in Figure 11. It can be seen that, due to the influence of tether tension on its vibration period, when tether sag increases, the frequency of first in-plane and out-of-plane modes increases.

3.4. Effect of Tether Length on Its Modal Damping Ratios. Effect of tether length on its damping ratios of the first mode (both in-plane and out-of-plane) are shown in Figure 12. It is shown that, when tether length increases, the damping ratios of first in-plane and out-of-plane modes increase. However, the curve of the first in-plane modal damping ratio is nonlinear, whereas that of the first out-of-plane modal damping ratio is linear.

Effect of tether length on its frequency of the first mode is shown in Figure 13. It is shown that, when tether length increases, the frequency of first in-plane and out-of-plane modes decrease.

\section{Conclusions}

(1) The change of inclination leads to the change of sag. The initial configuration of sag is symmetric. Thus, the sag only affects the damping ratio of the inner symmetric mode, namely, that of the first and the third mode. The sag has no effect on the second and forth order modes which are antisymmetric modes. Therefore, the sag is only existed in-plane and has no effect on the out-of-plane mode.

(2) The first and the third in-plane modal damping ratios of tether are in direct proportion to their 
inclination, whereas in inverse proportion to their sag.

(3) The first modal damping ratio of tether (both inplane and out-of-plane) is in direct proportion to its length, whereas in inverse proportion to its initial tension.

\section{Data Availability}

The data used to support the findings of this study are available from the corresponding author upon request.

\section{Conflicts of Interest}

The authors declare that there are no conflicts of interest regarding the publication of this paper.

\section{Acknowledgments}

This work was financially supported by the Research Fund of Liaocheng University (Grant no. 318011916) and the Natural Science Foundation of Shandong Province (Grant no. ZR2018BEE046).

\section{References}

[1] K. Q. Zhang, "Structural design and analysis of submerged floating tunnel and its health monitoring," Master Thesis, Zhejiang University, 2011.

[2] R. Svein, J. L. Bernt, M. O. Knut, M. M. Kjell, and H. Terje, "Dynamic response and fluid/structure interaction of submerged floating tunnels," Computers and Structures, vol. 72, pp. 659-685, 1999.

[3] J. Y. Chen, B. G. Wang, and S. N. Sun, "Analysis of vortexinduced dynamic response for the anchor cable of submerged floating tunnel," Chinese Journal of Engineering Mechanics, vol. 24, no. 10, pp. 186-192, 2007.

[4] S. N. Sun, J. Y. Chen, and J. Li, "Non-linear response of tethers subjected to parametric excitation in submerged floating tunnels," China Ocean Engineering, vol. 23, no. 1, pp. 167-174, 2009.

[5] Z. B. Su and S. N. Sun, "Non-linear free vibration of submerged floating tunnel tether," Applied Mechanics and Materials, vol. 580-583, pp. 1388-1391, 2014.

[6] F. Ge, M. S. Dong, L. Hui, and Y. S. Hong, "Vortex-induced vibration of submerged floating tunnel tethers under wave and current effects," Chinese Journal of Engineering $\mathrm{Me}$ chanics, vol. 23, no. S1, pp. 217-221, 2006.

[7] J. Y. Chen, S. N. Sun, and B. G. Wang, "Dynamic analysis for the tether of submerged floating tunnel," Chinese Journal of Computational Mechanics, vol. 25, no. 4, pp. 488-493, 2008.

[8] F. Ge, X. Long, L. Wang, and Y. S. Hong, "Study of vortexinduced vibration of submerged floating tunnel tube-tether coupled model," China Journal of Highway and Transport, vol. 22, no. 3, pp. 83-88+100, 2009.

[9] G. Luo, Y. Y. Shi, Q. Shen, and H. Li, "Lateral dynamic characteristics analysis for cable of submerged floating tunnel in water," Chinese Journal of Changan University, vol. 32, no. 3, pp. 73-78, 2012.

[10] Y. Q. Xiang and C. F. Chao, "Vortex-induced dynamic response for combined action of tube and cable of submerged floating tunnel," Chinese Journal of Zhejiang University, vol. 46, no. 3, pp. 409-415, 2012.
[11] D. I. Caruntu, "On nonlinear forced response of nonuniform beams," in Proceedings of the ASME Dynamic Systems and Control Conference, part $A$ and B, pp. 403-408, June 2008, https://asmedigitalcollection.asme.org/DSCC/proceedingsabstract/DSCC2008/43352/403/334059.

[12] M. Arefi and A. M. Zenkour, "Nonlocal electro-thermomechanical analysis of a sandwich nanoplate containing a Kelvin-Voigt viscoelastic nanoplate and two piezoelectric layers," Acta Mechanica, vol. 228, no. 2, pp. 475-493, 2017.

[13] S. Guo and S. Yang, "Free longitudinal vibrations of nonuniform rods," Science China Technological Sciences, vol. 54, no. 10, p. 2735, 2011.

[14] M. Arefi and A. M. Zenkour, "Wave propagation analysis of a functionally graded magneto-electro-elastic nanobeam rest on Visco-Pasternak foundation," Mechanics Research Communications, vol. 79, pp. 51-62, 2017.

[15] S. Lenci, F. Clementi, and C. E. N. Mazzilli, "Simple formulas for the natural frequencies of non-uniform cables and beams," International Journal of Mechanical Sciences, vol. 77, p. 155, 2013.

[16] M. Arefi and A. M. Zenkour, "Size-dependent free vibration and dynamic analyses of piezo-electro-magnetic sandwich nanoplates resting on viscoelastic foundation," Physica B: Condensed Matter, vol. 521, pp. 188-197, 2017.

[17] D. V. Bambill, C. A. Rossit, R. E. Rossi, D. H. Felix, and A. R. Ratazzi, "Transverse free vibration of non uniform rotating Timoshenko beams with elastically clamped boundary conditions," Meccanica, vol. 48, no. 6, p. 1289, 2013.

[18] A. M. Zenkour and M. Arefi, "Nonlocal transient electrothermomechanical vibration and bending analysis of a functionally graded piezoelectric single-layered nanosheet rest on visco-Pasternak foundation," Journal of Thermal Stresses, vol. 40, no. 2, pp. 167-184, 2017.

[19] E. Babilio, "Dynamics of an axially functionally graded beam under axial load," The European Physical Journal Special Topics, vol. 222, no. 7, p. 1519, 2013.

[20] M. Arefi and A. M. Zenkour, "Analysis of wave propagation in a functionally graded nanobeam resting on visco-Pasternak's foundation," Theoretical and Applied Mechanics Letters, vol. 7, no. 3, pp. 145-151, 2017.

[21] F. Clementi, L. Demeio, C. E. N. Mazzilli, and S. Lenci, "Nonlinear vibrations of non-uniform beams by the MTS asymptotic expansion method," Continuum Mechanics and Thermodynamics, vol. 27, no. 4-5, p. 703, 2015.

[22] M. Arefi and A. M. Zenkour, "Vibration and bending analyses of magneto-electro-thermo-elastic sandwich microplates resting on viscoelastic foundation," Applied Physics A, vol. 123, no. 8, p. 550, 2017.

[23] E. Babilio, "Dynamics of functionally graded beams on viscoelastic foundation," International Journal of Structural Stability and Dynamics, vol. 14, no. 8, Article ID 1440014, 2014.

[24] Y. Q. Ni, W. J. Lou, and J. M. Ko, "A hybrid pseudo-force/ laplace transform method for non-linear transient response of a suspended cable," Journal of Sound and Vibration, vol. 238, no. 2, pp. 189-214, 2000.

[25] S.-N. Sun and Z.-B. Su, "Parametric vibration of submerged floating tunnel tether under random excitation," China Ocean Engineering, vol. 25, no. 2, p. 349, 2011.

[26] H. L. Ma, "Vibration control of offshore platform with viscoelastic dampers," Master Thesis, Ocean University of China, Qingdao, China, 2003.

[27] H. M. Irvine, Cable Structures, Institute of Architectural and Civil Engineering, Cambridge, MA, USA, 1981. 